\title{
Boundary control of a rotating Timoshenko beam
}

\author{
Stephen W. Taylor* Stephen C. B. Yau ${ }^{\dagger}$
}

(Received 2 February 2002)

\begin{abstract}
The boundary control of a rotating beam is investigated. The beam is modelled by the Timoshenko beam equations, which are a system of two coupled wave equations that include the effects of shearing and the rotational inertia of cross-sections of the beam. The beam, which is pivoted at one end and free at the other, has physical parameters that may vary along the length of the beam. Conditions are found for which both the angle of rotation and the vibrations of the beam may be controlled by applying a force at the free end and a torque at the pivoted end. This is an improvement on previous work of the first author, who showed only that the vibrations may be controlled.
\end{abstract}

*Department of Mathematics, University of Auckland, New ZEALAnd. mailto:s.taylor@auckland.ac.nz

${ }^{\dagger}$ Department of Mathematics, University of Auckland, NEw ZEALAND. mailto:stephen@seito.com.hk

${ }^{0}$ See http: //anziamj . austms .org. au/V44/E033 for this article, (c) Austral. Mathematical Soc. 2003. Published January 29, 2003. ISSN 1446-8735 


\section{Contents}

1 Introduction

E144

2 Timoshenko Beam Theory $\quad$ E147

2.1 Basic Beam Theory . . . . . . . . . . . . . E147

2.2 Characteristics of the beam . . . . . . . E150

2.3 Propagation of singularities . . . . . . . . E E153

2.4 Ratio of Moduli and Wave Speeds . . . . . . . . E155

2.5 Boundary Conditions . . . . . . . . . . E E156

2.6 Boundary Controllability of the non-Rotating Beams E158

2.7 Existence of Solutions to the Beam Equations . . E159

3 A Rotating Timoshenko Beam

E163

3.1 Relative Motion of the Rotating Beam . . . . . E163

3.2 Energy and Equations of a Rotating Beam . . . . E166

3.3 Partial Boundary Controllability of the Rotating Beam E170

3.4 Existence of Solutions to the Beam Equations . . E172

4 An Auxiliary Problem and Boundary Controllability

E173

4.1 Auxiliary Problem and Contraction Properties . . E173

4.2 Controlling the Hinged Beam . . . . . . . . . E178

4.3 Controlling the Rotating Beam . . . . . . . E180

References

E183

\section{Introduction}

Vibration has long been known for its capacity for disturbance, discomfort, damage and destruction. Since ancient times, mankind has tried to investigate ways to control this phenomenon. Thus, in the 
development of control theory for partial differential equations over the last few decades, it is not surprising that fundamental elastic systems such as beams have received a lot of attention. The familiar Euler-Bernoulli beam equation has been the subject of many investigations. In its simplest form this models the beam's transverse vibrations by the equation

$$
\rho A W_{T T}+E I W_{X X X X}=0 .
$$

Here the $X$-axis coincides with the beam when it is at rest and $W(X, T)$ is the displacement of the beam at time $T$ in a direction that is perpendicular to the $X$-axis. The parameters appearing here are the beam's density as mass per unit volume $\rho$, Young's modulus of elasticity $E$, cross-sectional area $A$, and moment of inertia $I$.

The Timoshenko beam theory has also received some attention. This theory is an improvement of the Euler-Bernoulli system in that it also takes into account rotational inertia and the shearing deformation that occurs within a beam as it vibrates. The model involves two coupled wave equations

$$
\begin{aligned}
\rho A W_{T T}+\left(k A G\left(\Psi-W_{X}\right)\right)_{X} & =0, \\
\rho I \Psi_{T T}-\left(E I \Psi_{X}\right)_{X}+k A G\left(\Psi-W_{X}\right) & =0 .
\end{aligned}
$$

Here $W$ and $\Psi$ are the transverse and angular displacements respectively. This model is described in detail in the next section.

One way to impose controls for partial differential equations (PDEs) is through boundary conditions. The space-variable domain for a beam of length $L$ may be taken to be the interval $[0, L]$ which has boundary points $X=0$ and $X=L$. Various boundary conditions are possible. For instance, if the beam is hinged at the origin 
and free at its other end then the boundary conditions take the form

$$
\begin{aligned}
W(0, T) & =0, \\
E I(0) \Psi_{X}(0, T) & =-\tau(T), \\
\Psi_{X}(L, T) & =0, \\
k A G(L)\left(\Psi(L, T)-W_{X}(L, T)\right) & =-f(T) .
\end{aligned}
$$

Here $\tau$ is an external torque applied at the origin and $f$ is a force applied at the free end. Such boundary conditions are described in greater detail in the next section. Null boundary controllability concerns finding functions $f$ and $\tau$ that drive solutions of the PDE to its zero displacement rest state during a specified time interval. The functions $f$ and $\tau$ are often called controls or control functions. In fact, for linear systems such as this, null-controllability implies that initial states can be driven to any specified state.

The Timoshenko system is hyperbolic and consequently disturbances move with finite speed along the beam. Because of this it is impossible to control the Timoshenko beam within an indefinitely small time interval. In fact there is a time $T_{0}$ associated with the speed of propagation of singularities (see the next section for a description of this) such that the beam is not controllable during time intervals $[0, t]$ if $t<T_{0}$. It is interesting to note that boundary controllability of the Euler-Bernoulli beam equation is possible for arbitrarily small time intervals.

The boundary controllability of hinged-free and clamped-free Timoshenko beams is investigated in [6]. The hinged-free case is more closely related to the case of a rotating beam, so we briefly discuss its controllability here. Provided that the control time is chosen to be greater than $T_{0}$, it is shown in [6] that the system may be driven to one of its rest states if and only if there are no nontrivial solutions of an overdetermined eigenvalue problem consisting of the 
DES

$$
\begin{aligned}
\mu^{2} \rho W-\left(k A G\left(\Psi-W_{X}\right)\right)_{X} & =0 \\
\mu^{2} \rho I \Psi+\left(E I \Psi_{X}\right)_{X}-k A G\left(\Psi-W_{X}\right) & =0
\end{aligned}
$$

and the six homogeneous boundary conditions

$$
\begin{aligned}
W(0)=0, \quad \Psi(0) & =0, \quad \Psi_{X}(0)=0, \\
W(L)=0, \quad W_{X}(L)-\Psi(L) & =0, \quad \Psi_{X}(L)=0 .
\end{aligned}
$$

It is shown in [6] that nontrivial solutions of this system exist for certain values of the elastic parameters.

One shortfall of the theory developed in [6] is the fact that the hinged-free system has many rest states $\Psi(X)=\theta_{0}, W(X)=\theta_{0} X$. A further shortfall is that the hinged-free beam is a simplistic model of a rotating beam that allows for only small angular displacements.

The two main aims of this paper are to overcome these two shortfalls. In fact in Section 3 we develop a better model for a rotating Timoshenko beam which allows for larger angular displacements and in Section 4 we modify our method to obtain controllability for the $\theta_{0}=0$ case as well. A further aim is to correlate existing information on the Timoshenko Theory.

\section{Timoshenko Beam Theory}

\subsection{Basic Beam Theory}

The Timoshenko theory (or thick beam theory) accounts for both the effect of rotary inertia and shear deformation, which are neglected when applied to Euler-Bernoulli beam theory (or thin beam 
theory). The transverse vibration of the beam depends on its geometrical and material properties as well as the external applied force and torque. The geometrical properties refer mainly to its length $L$, size and shape of its cross-section such as its area $A$, moment of inertia $I$ with respect to the central axis of bending, and Timoshenko's shear coefficient $k$ which is a modifying factor $(k<1)$ to account for the distribution of shearing stress such that effective shear area is equal to $k A$. The material properties refer to its density $\rho$ in mass per unit volume, Young's modulus or modulus of elasticity $E$ and shear modulus or modulus of rigidity $G$. We assume that $\rho, E, G$, $k, A$ and $I$ are all positive, $C^{2}$ functions of the space variable.

A differential element of a beam is shown in Figure 1 . Here $W$ is the transverse displacement of the neutral line at a distance $X$ from the left end of the beam at time $T$. Due to the effect of shear, the original rectangular element changes its shape to somewhat like a parallelogram with its sides slightly curved.

The shear angle $\vartheta$ (or loss of slope) is now equal to the slope of bending $\Psi$ less slope of centerline $W_{X}$ in the form

$$
\vartheta=\Psi-W_{X},
$$

and the shear force $Q$ is against the internal shear loading in the form

$$
Q=-k A G \vartheta=-k A G\left(\Psi-W_{X}\right) .
$$

Similarly, the bending moment $M$ is against the internal elastic inertia in the form

$$
M=-E I \Psi_{X} .
$$

Moreover, from Figure 1, we equate the transverse force and rotary inertia of the element to form the following four simultaneous PDEs 


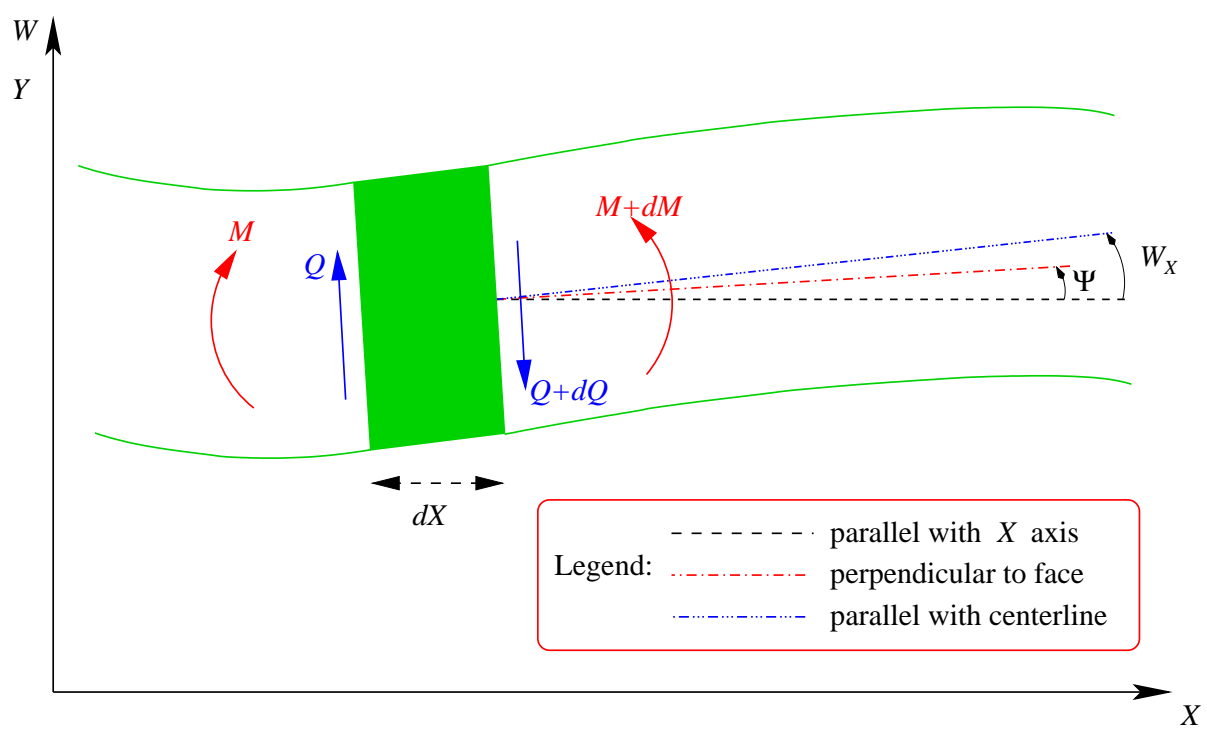

Figure 1: Differential analysis of Timoshenko beam

$$
\begin{aligned}
M+E I \Psi_{X} & =0 \\
Q+k A G\left(\Psi-W_{X}\right) & =0 \\
M_{X}-Q+\rho I \Psi_{T T} & =0 \\
Q_{X}-\rho A W_{T T} & =0 .
\end{aligned}
$$

Further, Equations (1a) and (1c) involve rotational motion while Equations (1b) and (1d) involve transverse motion of the element.

Eliminating $M$ and $Q$ from (1) yields two simultaneous PDEs in $W$ and $\Psi$ :

$$
\begin{aligned}
\rho A W_{T T}+\left(k A G\left(\Psi-W_{X}\right)\right)_{X} & =0, \\
\rho I \Psi_{T T}-\left(E I \Psi_{X}\right)_{X}+k A G\left(\Psi-W_{X}\right) & =0 .
\end{aligned}
$$


Equation (2a) is an equilibrium of translational force per unit length against the internal shear force gradient while Equation (2b) is an equilibrium of rotational torque per unit length equating to the gradient of internal bending moment against the internal shear force. This form is convenient for finding the normal modes and frequency of free vibration and the solution is in the form of $(W, \Psi)$.

In the case of a uniform beam, $\Psi$ can be eliminated from the above two equations to form a single equation

$$
\frac{E I}{\rho A} W_{X X X X}-\frac{I}{A}\left(\frac{E}{k G}+1\right) W_{X X T T}+\frac{\rho I}{k G A} W_{T T T T}+W_{T T}=0 .
$$

This equation has four terms in the unit of force per unit mass or acceleration. They are terms involving bending moment, shear force, rotational motion and translational motion respectively. When the shear and rotational terms are small and disregarded, the equation will be that of the Euler-Bernoulli beam.

\subsection{Characteristics of the beam}

We differentiate (1a) and (1b) with respect to time $T$ and introduce new variables, linear velocity $\mathcal{V}$ and angular velocity $\Omega$ to yield

$$
\begin{aligned}
\Omega_{X}+\frac{M_{T}}{E I} & =0, \\
\mathcal{V}_{X}-\frac{Q_{T}}{k A G}-\Omega & =0, \\
M_{X}-Q+\rho I \Omega_{T} & =0, \\
Q_{X}-\rho A \mathcal{V}_{T} & =0 .
\end{aligned}
$$

A linear combination of Equations (4a) and (4c) yields

$$
\alpha\left(M_{T}+E I \Omega_{X}\right)+M_{X}+\rho I \Omega_{T}-Q=0,
$$


where $\alpha$ can be determined in such a way that the partial derivatives of the above equation combine to give total derivatives $\frac{d M}{d X}$ and $\frac{d \Omega}{d X}$ in the direction of unknown characteristic lines.

From $\frac{d M}{d X}=M_{X}+M_{T} \frac{d T}{d X}$, we have $\alpha=\frac{d T}{d X}$ and from $\frac{d \Omega}{d X}=$ $\Omega_{X}+\Omega_{T} \frac{d T}{d X}$, we have $\alpha=\frac{\rho}{E} / \frac{d T}{d X}$. Since the slope of characteristics must be the same in both cases

$$
\begin{gathered}
\alpha^{2}=\frac{\rho}{E} \\
\alpha=\frac{d T}{d X}= \pm \sqrt{\frac{\rho}{E}}= \pm \frac{1}{v_{2}}
\end{gathered}
$$

where $v_{2}=\sqrt{E / \rho}$. Substituting these equations back into (5) and multiplying by $d T$ yields

$$
\pm \frac{1}{v_{2}} d M+\rho I d \Omega-Q d T=0
$$

where $d T= \pm \frac{1}{v_{2}} d X$.

Similarly, a linear combination of Equations (4b) and (4d) yields

$$
\pm \frac{1}{v_{1}} d Q-\rho A d \mathcal{V} \pm \frac{k A G}{v_{1}} \Omega d T=0
$$

where $v_{1}=\sqrt{k G / \rho}$ and $d T= \pm \frac{1}{v_{1}} d X$, hence the system of Equations (4) is hyperbolic and associated with it are the four real characteristic equations:

$$
\begin{aligned}
I^{+} \text {and } I^{-}, & \frac{d T}{d X}= \pm \frac{1}{v_{2}} \\
I I^{+} \text {and } I I^{-}, & \frac{d T}{d X}= \pm \frac{1}{v_{1}} .
\end{aligned}
$$

Figure 2 illustrates these four characteristics, $I^{+}, I^{-}$and $I I^{+}, I I^{-}$ passing through a point $P$ in the space-time plane. By properties of 


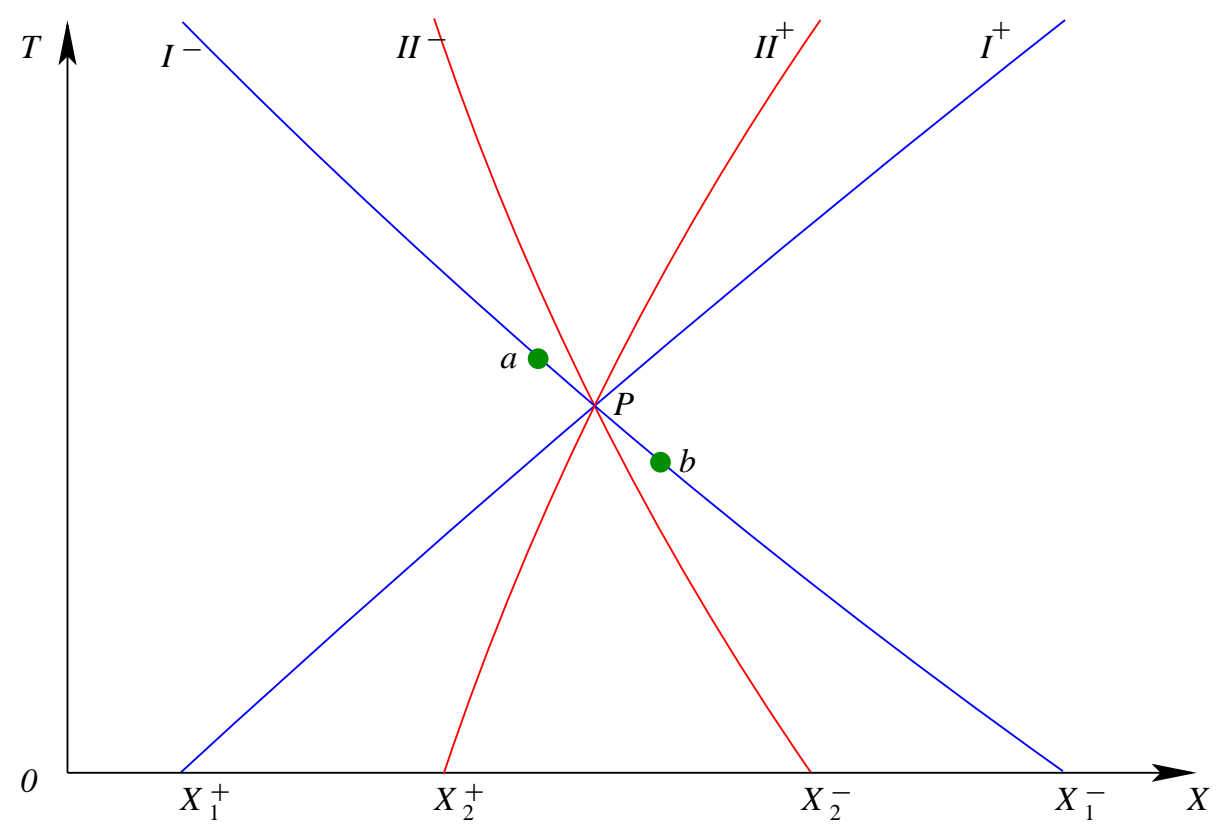

Figure 2: The characteristics of Timoshenko equations through point $P$ 
characteristics, the values of the unknowns $M, Q, \Omega$ and $\mathcal{V}$ at the point $P$ depend only on their initial values on the $X$-axis, between $X_{1}^{+}$and $X_{1}^{-}$for characteristics $I^{+}$and $I^{-}$, and between $X_{2}^{+}$and $X_{2}^{-}$ for characteristics $I I^{+}$and $I I^{-}$. Furthermore, these values at $P$ can, in turn, have influence only on points lying in the region above $P$ enveloped by the $I^{+}$and $I^{-}$characteristics through $P$. Thus no signal can proceed along the beam with a velocity greater than $v_{2}$. In contrast, the Euler-Bernoulli beam theory predicts incorrectly that disturbances propagate with infinite velocity.

For easy reference, the four characteristic differential equations are grouped below:

$$
\begin{array}{cl}
\text { along } I^{+}, I^{-}: & \pm \frac{1}{v_{2}} d M+\rho I d \Omega-Q d T=0 ; \\
\text { along } I I^{+}, I I^{-}: & \pm \frac{1}{v_{1}} d Q-\rho A d \mathcal{V} \pm \rho A v_{1} \Omega d T=0 .
\end{array}
$$

\subsection{Propagation of singularities}

Characteristics are lines across which singularities may exist. In Figure 2, singularities or jumps in $M$ and $\Omega$ can therefore exist across the $I^{+}$and $I^{-}$characteristics and propagate with velocity $v_{2}$, while singularities in $Q$ and $V$ can exist across the $I^{+}$and $I^{-}$ characteristics and propagate with velocity $v_{1}$.

Let $a$ and $b$ be two points on $I^{-}$across point $P$ and very close to $P$ as shown in Figure 2. Assuming instantaneous loading, if $M$ is discontinuous across $P$, a finite value of moment $\delta M=M_{a}-M_{b}$ is allowed to approach $P$ from either side. From Equation (7a), $d T$ approaches zero because $a$ and $b$ are very close, so

$$
\delta M=v_{2} \rho I \delta \Omega .
$$


Thus, such jumps $\delta M$ and $\delta \Omega$ across $P$ will travel along characteristic $I^{+}$at speed $v_{2}$. Similarly, the jumps on $I^{+}$across $P$ will travel along $I^{-}$at speed $v_{2}$ when passing through $P$. Applying the same theory, similar results can be obtained with jumps $\delta Q$ and $\delta \mathcal{V}$ for $I I$ across $P$ with speed $v_{1}$.

Taking account of the direction of the characteristics, we have:

$$
\begin{aligned}
\text { along } I^{+}, I^{-}: & \delta M= \pm v_{2} \rho I \delta \Omega ; \\
\text { along } I I^{+}, I I^{-}: & \delta Q=\mp v_{1} \rho A \delta \mathcal{V} .
\end{aligned}
$$

Hence a jump in $M$ on $I^{+}$or $I^{-}$is always accompanied by a definite jump in $\Omega$. Similarly $Q$ and $\mathcal{V}$ are coupled together.

From (7a) along $I^{+}$, since $Q$ is continuous across $I^{+}$, the difference across $P$ gives

$$
d(\delta M)+v_{2} \rho I d(\delta \Omega)=0,
$$

Eliminating $\delta \Omega$ from (8a),

$$
d(\delta M)+v_{2} \rho I d\left(\frac{\delta M}{v_{2} \rho I}\right)=0 .
$$

which can be integrated for a jump from point 1 to point 2 to give

$$
(\delta M)_{2}=(\delta M)_{1} \sqrt{\frac{\left(v_{2} \rho I\right)_{2}}{\left(v_{2} \rho I\right)_{1}}} .
$$

Similarly, we eliminate $\delta M$ from (8a) to give

$$
(\delta \Omega)_{2}=(\delta \Omega)_{1} \sqrt{\frac{\left(v_{2} \rho I\right)_{1}}{\left(v_{2} \rho I\right)_{2}}} .
$$

It can be shown that the identical relationship holds between jumps in $M$ or 2 similar points on $I^{-}$characteristics. 
Applying the same theory to $I I$ yields

$$
\begin{aligned}
& (\delta Q)_{2}=(\delta Q)_{1} \sqrt{\frac{\left(v_{1} \rho A\right)_{2}}{\left(v_{1} \rho A\right)_{1}}} \\
& (\delta \mathcal{V})_{2}=(\delta \mathcal{V})_{1} \sqrt{\frac{\left(v_{1} \rho A\right)_{1}}{\left(v_{1} \rho A\right)_{2}}}
\end{aligned}
$$

The interested reader may refer to John [2, p.35-37] or Leonard and Budiansky [3] for more details for propagation of singularities and travelling waves in beams.

\subsection{Ratio of Moduli and Wave Speeds}

Hooke's law states that the uniaxial stress $\sigma_{X}$ (or axial force per unit sectional area) applied to a bar in the $X$ direction is proportional to the strain $\epsilon_{X}$ (or elongation per unit length) within the elastic limit in the form

$$
E=\frac{\sigma_{X}}{\epsilon_{X}},
$$

where the constant $E$ is the modulus of elasticity. In the case of 3dimensions, if a bar is lengthened by an axial force along the $X$-axis, there is always a corresponding reduction of length in the $Y$ and $Z$ directions and a ratio known as Poisson's ratio $\nu(0<\nu<1)$ is introduced. This ratio refers to the strains in these directions and is a constant for stresses within the elastic limit defined by

$$
\nu=-\frac{\epsilon_{Y}}{\epsilon_{X}}=-\frac{\epsilon_{Z}}{\epsilon_{X}}
$$

where $\epsilon_{X}$ is the strain due only to the axial force in the $X$ direction, and $\epsilon_{Y}$ and $\epsilon_{Z}$ are the strains induced in the $Y$ and $Z$ directions 
respectively. The minus sign indicates a decrease in transverse dimensions when $\epsilon_{X}$ is positive, as in the case of tensile elongation.

$$
\frac{G}{E}=\frac{1}{2(1+\nu)} .
$$

Proof of this formula and further details can be obtained from most Mechanics or Strength of Material books such as [5] Pages 74-84.

Hence the ratio of wave speeds $V$ can be derived directly from $k$ and $\nu$ as

$$
V=\frac{v_{1}}{v_{2}}=\sqrt{\frac{k G}{E}}=\sqrt{\frac{k}{2(1+\nu)}}<\frac{1}{\sqrt{2}} \approx 0.7071 .
$$

So in practice, $G, E, v_{1}$ and $v_{2}$ are all different such that $2 G<E$ and $\sqrt{2} v_{1}<v_{2}$. Hence the characteristics are distinct.

Common values of Poisson's ratio $\nu$ are 0.25 to 0.30 for steel, approximately 0.33 for most other metals, and 0.20 for concrete [5, p.43].

The Timoshenko shear coefficient $k$ is derived by Mindlin and Deresiewics [4] to be $\pi^{2} / 12 \approx 0.822$ for rectangular cross-section and 0.847 for circular cross-section.

\subsection{Boundary Conditions}

In general, there are three common types of boundary conditions at each end of the beam. They are hinged, clamped and free type, again involving dynamics and geometrical properties of the beam:

hinged type $W=0, \quad M=E I \Psi_{X}=0 ;$ 
clamped type $W=0, \quad \Psi=0 ;$

free type $Q=k A G\left(\Psi-W_{X}\right)=0, \quad M=E I \Psi_{X}=0$.

The combination of the above at both ends of the beam yields six situations: hinged-free, clamped-free, hinged-clamped, hinged-hinged, clamped-clamped, and free-free types.

In this section we investigate free vibration and controls mainly on the hinged-free type (for convenience, hereafter we call it a hinged beam). Also we study the clamped-free type (hereafter called a clamped beam), which has a similar set of equations to the hinged beam, and use it for comparison of equations and as a test problem to test numerical code.

The control functions of the hinged beam with one end hinged at the origin and the other end free, are a torque $\tau$ applied at the origin and a force $f$ applied at the free end. The associated boundary conditions are

$$
\begin{aligned}
W(0, T) & =0, \\
E I(0) \Psi_{X}(0, T) & =-\tau(T), \\
\Psi_{X}(L, T) & =0, \\
k A G(L)\left(\Psi(L, T)-W_{X}(L, T)\right) & =-f(T) .
\end{aligned}
$$

For the clamped beam with one end clamped at the origin and the other end free, the control functions are a force $f$ and a torque $\tau$ applied at the free end. The associated boundary conditions for this case are

$$
\begin{aligned}
W(0, T) & =0, \\
\Psi(0, T) & =0, \\
k A G(L)\left(\Psi(L, T)-W_{X}(L, T)\right) & =-f(T), \\
E I(L) \Psi_{X}(L, T) & =-\tau(T) .
\end{aligned}
$$


In each case, the system is completed by including the initial conditions

$$
\begin{aligned}
W(X, 0)=W^{o}(X), & W_{T}(X, 0)=\mathcal{V}^{o}(X), \\
\Psi(X, 0)=\Psi^{o}(X), & \Psi_{T}(X, 0)=\Omega^{o}(X) .
\end{aligned}
$$

\subsection{Boundary Controllability of the non-Rotating Beams}

Let $T_{1}$ and $T_{2}$ denote the times required for the two types of waves to travel along the whole length of the hinged beam

$$
T_{1}=\int_{0}^{L} \frac{d X}{v_{1}(X)}, \quad T_{2}=\int_{0}^{L} \frac{d X}{v_{2}(X)},
$$

and $T_{o}=2 \max \left(T_{1}, T_{2}\right)$, and suppose that $\mathcal{T}>T_{o}$. We seek control functions $f$ and $\tau$ belonging to $L^{2}(0, \mathcal{T})$ for the hinged beam that drives the solutions to one of the states

$$
W_{T}(X, \mathcal{T})=\Psi_{T}(X, \mathcal{T})=0, \Psi(X, \mathcal{T})=\theta_{o}, W(X, \mathcal{T})=\theta_{o} X
$$

where $\theta_{o}$ is a constant that can be interpreted as the weighted average angle of rotation of the beam about the point at $X=0$.

For the clamped beam, solutions are driven to the states

$$
W(X, \mathcal{T})=\Psi(X, \mathcal{T})=W_{T}(X, \mathcal{T})=\Psi_{T}(X, \mathcal{T})=0 .
$$

In [6] it is shown that there are certain over-determined eigenvalue problems associated with the hinged beam and the clamped beam. We discuss these here and that of a rotating beam in Section 4. The controllability of each of these two beam systems is linked to the non-existence of an eigenfunction, and uncontrollability is linked 
to the existence of such an eigenfunction. For this reason, we call such eigenvalue problems controllability eigenvalue problems. Here, each eigenvalue problem with eigenvalue parameter $\mu$ consists of the ordinary differential equations

$$
\begin{aligned}
\mu^{2} \rho W-\left(k A G\left(\Psi-W_{X}\right)\right)_{X} & =0, \\
\mu^{2} \rho I \Psi+\left(E I \Psi_{X}\right)_{X}-k A G\left(\Psi-W_{X}\right) & =0,
\end{aligned}
$$

and six homogeneous boundary conditions. The boundary conditions associated with the eigenvalue problem for the hinged beam are

$$
\begin{aligned}
W(0)=0, \quad \Psi(0)=0, & \Psi_{X}(0)=0, \\
W(L)=0, \quad W_{X}(L)-\Psi(L)=0, & \Psi_{X}(L)=0 .
\end{aligned}
$$

The boundary conditions associated with the eigenvalue problem for the clamped beam are

$$
\begin{array}{rrrl}
W(0)=0, & W(L)=0, & W_{X}(L)=0, \\
\Psi(0)=0, & \Psi(L)=0, & \Psi_{X}(L)=0 .
\end{array}
$$

The eigenvalue problem for the clamped beam has only trivial solutions (one need consider only the boundary conditions at $X=L$ to see this), so it is controllable.

\subsection{Existence of Solutions to the Beam Equations}

To outline the existence theory of each of the systems (2), (11), (13) and the systems (2), (12), (13), we use the classical method of characteristics approach as used in Section 2.2. In this section, we 
assume that $\rho, E, G, k, A$ and $I$ are all positive, $C^{1}$ functions of the space variable.

The analysis is simplified by introducing the column vector $\mathbf{U}=$ $\left[U_{1}, U_{2}, U_{3}, U_{4}\right]^{T}$ where

$$
\begin{aligned}
& U_{1}=-\left(\sqrt{k A G}\left(\Psi-W_{X}\right)+\sqrt{\rho A} W_{T}\right) / 2, \\
& U_{2}=-\left(\sqrt{k A G}\left(\Psi-W_{X}\right)-\sqrt{\rho A} W_{T}\right) / 2, \\
& U_{3}=-\left(\sqrt{E I} \Psi_{X}-\sqrt{\rho I} \Psi_{T}\right) / 2, \\
& U_{4}=-\left(\sqrt{E I} \Psi_{X}+\sqrt{\rho I} \Psi_{T}\right) / 2,
\end{aligned}
$$

from which we see that

$$
\begin{aligned}
U_{2}+U_{1} & =-\sqrt{k A G}\left(\Psi-W_{X}\right), \\
U_{2}-U_{1} & =\sqrt{\rho A} W_{T}, \\
U_{4}+U_{3} & =-\sqrt{E I} \Psi_{X}, \\
U_{4}-U_{3} & =-\sqrt{\rho I} \Psi_{T} .
\end{aligned}
$$

The reason for coupling $U_{1}$ and $U_{2}$ together is that they are equations involving translational motion. Similarly, $U_{3}$ and $U_{4}$ are coupled together because they are equations involving rotational motion.

The beam equations (2) are thus transformed to a single vector equation

$$
\mathbf{U}_{T}+\Lambda \mathbf{U}_{X}=\mathbf{B U}-\Lambda_{X} \mathbf{U} / 2
$$

with

$$
\Lambda=\left[\begin{array}{llll}
v_{1} & & & \\
& -v_{1} & & \\
& & v_{2} & \\
& & & -v_{2}
\end{array}\right] \text { and } \mathbf{B}=\left[\begin{array}{rrrr}
0 & b_{1} & b_{2} & -b_{2} \\
-b_{1} & 0 & b_{2} & -b_{2} \\
-b_{2} & -b_{2} & 0 & b_{3} \\
b_{2} & b_{2} & -b_{3} & 0
\end{array}\right]
$$


where $\Lambda$ is a diagonal matrix, $v_{1}$ and $v_{2}$ are speeds of the characteristics derived in Section 2.2, and $B$ is a skew-symmetric matrix with

$$
\begin{aligned}
& b_{1}=\frac{1}{2}\left(\sqrt{k A G}\left(\frac{1}{\sqrt{\rho A}}\right)_{X}-\frac{(\sqrt{k A G})_{X}}{\sqrt{\rho A}}\right) \\
& b_{2}=-\frac{1}{2} \sqrt{\frac{k A G}{\rho I}} \\
& b_{3}=\frac{1}{2}\left(\sqrt{E I}\left(\frac{1}{\sqrt{\rho I}}\right)_{X}-\frac{(\sqrt{E I})_{X}}{\sqrt{\rho I}}\right) .
\end{aligned}
$$

The mechanical energy of the beam is

$$
\mathcal{E}=\frac{1}{2} \int_{0}^{L} k A G\left(\Psi-W_{X}\right)^{2}+\rho A W_{T}^{2}+E I \Psi_{X}^{2}+\rho I \Psi_{T}^{2} d X .
$$

In the new variables, the energy equation (21) is now in the form

$$
\mathcal{E}=\int_{0}^{L} U_{1}^{2}+U_{2}^{2}+U_{3}^{2}+U_{4}^{2} d X
$$

Boundary conditions (11) for the hinged beam now take the form

$$
\begin{aligned}
U_{2}(0, T)-U_{1}(0, T) & =0, \\
U_{4}(0, T)+U_{3}(0, T) & =(E I)(0)^{-1 / 2} \tau(T), \\
U_{4}(L, T)+U_{3}(L, T) & =0, \\
U_{2}(L, T)+U_{1}(L, T) & =(k A G)(L)^{-1 / 2} f(T) .
\end{aligned}
$$

Boundary conditions (12) for the clamped beam take the form

$$
\begin{aligned}
U_{2}(0, T)-U_{1}(0, T) & =0 \\
U_{4}(0, T)-U_{3}(0, T) & =0 \\
U_{2}(L, T)+U_{1}(L, T) & =(k A G)(L)^{-1 / 2} f(T), \\
U_{4}(L, T)+U_{3}(L, T) & =(E I)(L)^{-1 / 2} \tau(T) .
\end{aligned}
$$


In each case, the initial condition of the system can be denoted

$$
\mathbf{U}(X, 0)=\mathbf{g}(X) .
$$

We quote herewith the theorems of Classical and Finite Energy Solutions without proof from the paper [6].

Theorem 1 (Classical Solutions) If the boundary data $f, \tau$ and the initial data $\mathbf{g}$ are continuously differentiable and satisfy the appropriate compatibility conditions, then each of the systems (20), (23), (25) and that of (20), (24), (25) has a unique classical solution.

Theorem 2 (Finite Energy Solutions I) If the boundary data $f$, $\tau$ are in $L^{2}(0, \mathcal{T})$ and the initial data $\mathbf{g} \in \mathcal{H}$, then each of the systems (20), (23), (25) and that of (20), (24), (25) has a unique finite energy solution $\mathbf{U}$. In fact, $\mathbf{U} \in C(0, \mathcal{T} ; \mathcal{H})$.

Theorem 3 (Finite Energy Solutions II) If the boundary data $f$, $\tau$ are in $L^{2}(0, \mathcal{T})$ and $\left(W^{o}, \Psi^{o}\right) \in \mathcal{V}_{h}$ and $\left(\mathcal{V}^{o}, \Omega^{o}\right) \in \mathcal{H}_{o}$, then the system (2), (11), (25) has a unique weak solution $(W, \Psi)$ such that $(W, \Psi) \in C\left(0, \mathcal{T} ; \mathcal{V}_{h}\right),\left(W_{T}, \Psi_{T}\right) \in C\left(0, \mathcal{T} ; \mathcal{H}_{o}\right)$.

Here we define the finite energy space $\mathcal{H}=\left(L^{2}(0, L)\right)^{4}$, the norm of which is given by

$$
\|\mathbf{U}\|=\left(\int_{0}^{L}\left|U_{1}\right|^{2}+\left|U_{2}\right|^{2}+\left|U_{3}\right|^{2}+\left|U_{4}\right|^{2} d X\right)^{1 / 2}
$$

and say that a weak solution $\mathbf{U}$ is a finite energy solution if $\mathbf{U} \in$ $L^{\infty}(0, \mathcal{T} ; \mathcal{H})$. Also we set $\mathcal{H}_{o}=\left(L^{2}(0, L)\right)^{2}$ and $\mathcal{V}_{h}=\{(W, \Psi) \in$ $\left.\mathcal{H}^{1}(0, L)^{2}: W(0)=0\right\}$. Further we can state a theorem similar to Theorem 3 for (2), (12), (25) by replacing $\mathcal{V}_{h}$ with $\mathcal{V}_{c}$ with 
$\mathcal{V}_{c}=\left\{(W, \Psi) \in \mathcal{H}^{1}(0, L)^{2}: W(0)=\Psi(0)=0\right\}$ where $(W, \Psi) \in$ $C\left(0, \mathcal{T} ; \mathcal{V}_{c}\right)$.

We again call such solutions finite energy solutions. The interested reader may refer to paper [6] for proof. We will see that similar results hold for the rotating beam.

\section{A Rotating Timoshenko Beam}

\subsection{Relative Motion of the Rotating Beam}

Consider a beam rotating anticlockwise about the pinned point at the origin with its moving frame as indicated by $X-Y$ axes which is inclined at an angle $\theta$ relative to a fixed reference frame as indicated by $\tilde{X}-\tilde{Y}$ axes at time $T$. The centerline of the beam is more or less coincident with the $X$-axis. More precisely, $\theta$ is the weighted average angle of inclination through the origin to be defined by Equation (30) and shown in Figure 3. Here we assume that the speed of rotation is small so that any longitudinal elongation and stress of the beam due to rotation are small and may be neglected.

Let $\mathcal{P}=[X, W]^{T}$ be the position vector of a point $P$ on the centerline of the beam at a distance $X$ from the origin. The tangent vector $\frac{\partial \mathcal{P}}{\partial X}$ of the centerline at $P$ is inclined at an angle $\beta$ with the $X$-axis.

To avoid confusion and for our easy reference, we use notations $W, \Psi$ (and $\beta$ to be defined as below), and $X-Y$ axes in the moving frame as we have defined before as in Figure 1 and introduce the corresponding new notations $\tilde{W}, \tilde{\Psi}, \tilde{\beta}$ and $\tilde{X}-\tilde{Y}$ axes in 


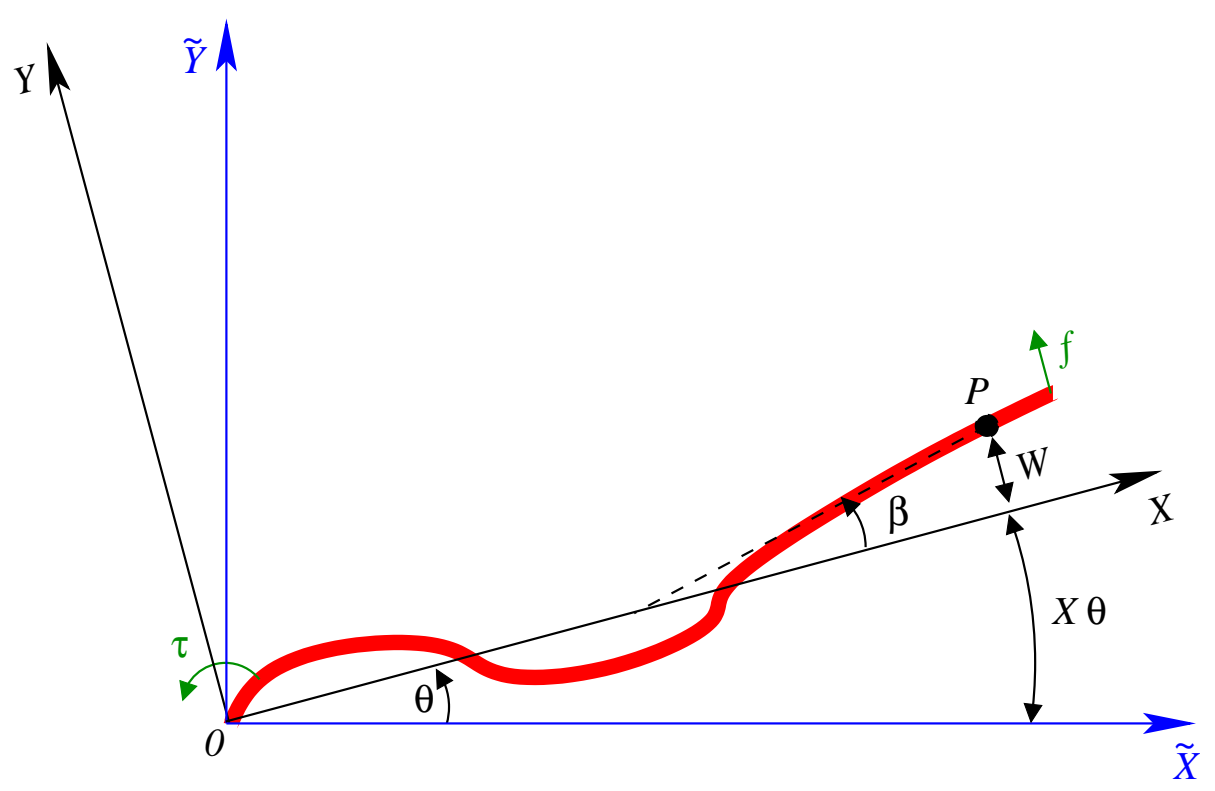

FIGURE 3: Rotating beam with moving frame

the fixed frame such that

$$
\begin{aligned}
\tilde{\Psi} & =\Psi+\theta, \\
\tilde{\beta} & =\beta+\theta .
\end{aligned}
$$

The transverse displacement $W$ is now in the form

$$
W=\int_{0}^{X} \beta(u) d u .
$$

The position vector with respect to the fixed reference frame is

$$
\mathcal{P}=\left[\begin{array}{rr}
\cos \theta & -\sin \theta \\
\sin \theta & \cos \theta
\end{array}\right]\left[\begin{array}{l}
X \\
W
\end{array}\right]=\left[\begin{array}{l}
X \cos \theta-W \sin \theta \\
X \sin \theta+W \cos \theta
\end{array}\right],
$$


hence the velocity vector $\mathcal{P}_{T}$ is given by

$$
\begin{aligned}
\mathcal{P}_{T} & =\left(X \theta_{T}+W_{T}\right)\left[\begin{array}{r}
-\sin \theta \\
\cos \theta
\end{array}\right]-W \theta_{T}\left[\begin{array}{c}
\cos \theta \\
\sin \theta
\end{array}\right] \\
& \approx\left(X \theta_{T}+W_{T}\right)\left[\begin{array}{r}
-\sin \theta \\
\cos \theta
\end{array}\right],
\end{aligned}
$$

and

$$
\left|\mathcal{P}_{T}\right|^{2} \approx\left(X \theta_{T}+W_{T}\right)^{2}=\tilde{W}_{T}^{2}
$$

where

$$
\tilde{W}=\int_{0}^{X} \tilde{\beta}(u) d u=W+X \theta .
$$

To define the angle $\theta$ we need to express the idea that a certain weighted average of the displacements is zero in the moving coordinate frame. We soon see that there are mathematical advantages to do this by requiring

$$
\int_{0}^{L} \rho I \Psi+\rho A X W d X=0,
$$

or

$$
\int_{0}^{L} \rho I(\tilde{\Psi}-\theta)+\rho A X \int_{0}^{X}(\tilde{\beta}(u)-\theta) d u d X=0,
$$

from which we define the weighted average angle $\theta$ as

$$
\theta=\frac{\int_{0}^{L} \rho I \tilde{\Psi}+\rho A X \tilde{W} d X}{\int_{0}^{L} \rho I+\rho A X^{2} d X} .
$$




\subsection{Energy and Equations of a Rotating Beam}

The kinetic energy of the beam is in the form

$$
\begin{aligned}
\mathrm{KE} & =\frac{1}{2} \int_{0}^{L} \rho I \tilde{\Psi}_{T}^{2}+\rho A \tilde{W}_{T}^{2} d X \\
& =\frac{1}{2} \int_{0}^{L} \rho I\left(\Psi_{T}+\theta_{T}\right)^{2}+\rho A\left(W_{T}+X \theta_{T}\right)^{2} d X \\
& =\frac{1}{2} \int_{0}^{L} \rho I \Psi_{T}^{2}+\rho A W_{T}^{2}+\rho\left(I+A X^{2}\right) \theta_{T}^{2} d X .
\end{aligned}
$$

The potential energy of the beam is now in the form

$$
\begin{aligned}
\mathrm{PE} & =\frac{1}{2} \int_{0}^{L} E I \tilde{\Psi}_{X}^{2}+k A G\left(\tilde{\Psi}-\tilde{W}_{X}\right)^{2} d X . \\
& =\frac{1}{2} \int_{0}^{L} E I \Psi_{X}^{2}+k A G\left(\Psi-W_{X}\right)^{2} d X .
\end{aligned}
$$

The virtual work functional subjected to constraint (29) is now in the form

$$
\mathcal{W}=\int_{0}^{T} \mathrm{KE}-\mathrm{PE}-\gamma(T) \int_{0}^{L} \rho I \Psi+\rho A X W d X d T
$$

where $\gamma(T)$ is the Lagrange multiplier associated with the constraint at time $T$. We calculate the first variation $\delta \mathcal{W}$ in order to apply the principle of virtual work and find the equations of motion. Applying 
integration by parts yields

$$
\begin{aligned}
\delta \mathcal{W}= & \int_{0}^{T} \int_{0}^{L} \rho I \Psi_{T} \delta \Psi_{T}+\rho A W_{T} \delta W_{T}+\rho\left(I+A X^{2}\right) \theta_{T} \delta \theta_{T} \\
& -E I \Psi_{X} \delta \Psi_{X}-k A G\left(\Psi-W_{X}\right)\left(\delta \Psi-\delta W_{X}\right) \\
& -\gamma(T)(\rho I \delta \Psi+\rho A X \delta W) d X d T \\
= & \int_{0}^{L}\left[\rho I \Psi_{T} \delta \Psi+\rho A W_{T} \delta W+\rho\left(I+A X^{2}\right) \theta_{T} \delta \theta\right]_{0}^{T} d X \\
& -\int_{0}^{T}\left[E I \Psi_{X} \delta \Psi-k A G\left(\Psi-W_{X}\right) \delta W\right]_{0}^{L} d T \\
& -\int_{0}^{T} \int_{0}^{L}\left(\rho I \Psi_{T T}-\left(E I \Psi_{X}\right)_{X}+k A G\left(\Psi-W_{X}\right)+\rho I \gamma(T)\right) \delta \Psi \\
& -\left(\rho A W_{T T}+\left(k A G\left(\Psi-W_{X}\right)\right)_{X}+\rho A X \gamma(T)\right) \delta W \\
& -\rho\left(I+A X^{2}\right) \theta_{T T} \delta \theta d X d T .
\end{aligned}
$$

For free vibration, and from Hamilton's principle for conservative systems as stated by Géradin and Rixen [1], $\delta \mathcal{W}=0$ so the coefficients of $\delta W, \delta \Psi$ and $\delta \theta$ must all be zero in the integrand and also at the end points.

Equating the coefficients of $\delta W, \delta \Psi$ and $\delta \theta$ yields

$$
\begin{aligned}
\rho A\left(W_{T T}+X \gamma(T)\right)+\left(k A G\left(\Psi-W_{X}\right)\right)_{X} & =0, \text { (31a) } \\
\rho I\left(\Psi_{T T}+\gamma(T)\right)-\left(E I \Psi_{X}\right)_{X}+k A G\left(\Psi-W_{X}\right) & =0, \text { (31b) } \\
\theta_{T T} \int_{0}^{L} \rho\left(I+A X^{2}\right) d X & =0 . \quad \text { (31c) }
\end{aligned}
$$

with homogeneous boundary conditions

$$
\Psi_{X}(0, T)=\Psi_{X}(L, T)=W(0, T)=\Psi(L, T)-W_{X}(L, T)=0 .
$$

Also from Equation (31c), since the moment of inertia at the origin $\int_{0}^{L} \rho\left(I+A X^{2}\right) d X>0$, the angular acceleration part $\theta_{T T}$ of 
the equation must vanish, which means that the beam rotates with constant angular velocity $\theta_{T}$ under free vibration.

When a torque $\tau(T)$ is applied at $X=0$ and a force $f(T)$ is applied at $X=L$, we must add the following term to the virtual work:

$$
\begin{aligned}
\delta \mathcal{W}^{+} & =\left.\int_{0}^{T} f \delta(\text { displacement })\right|_{X=L}+\left.\tau \delta(\text { angle })\right|_{X=0} d T \\
& =\int_{0}^{T} f(L \delta \theta+\delta W(L, T))+\tau(\delta \theta+\delta \Psi(0, T)) d T \\
& =\int_{0}^{T}(f L+\tau) \delta \theta+f \delta W(L, T)+\tau \delta \Psi(0, T) d T
\end{aligned}
$$

Equating the coefficient of $\delta \theta=0$ of the equation $\delta\left(\mathcal{W}+\mathcal{W}^{+}\right)=0$ yields

$$
\theta_{T T} \int_{0}^{L} \rho\left(I+A X^{2}\right) d X=f(T) L+\tau(T) .
$$

The associated boundary conditions are

$$
\begin{aligned}
W(0, T) & =0, \\
E I(0) \Psi_{X}(0, T) & =-\tau(T), \\
\Psi_{X}(L, T) & =0, \\
k A G(L)\left(\Psi(L, T)-W_{X}(L, T)\right) & =-f(T) .
\end{aligned}
$$

Taking second derivatives of Equation (29) and substituting (31), 
(32) and (33) yields

$$
\begin{aligned}
0= & \frac{d^{2}}{d T^{2}} \int_{0}^{L} \rho I \Psi+\rho A X W d X \\
= & \int_{0}^{L}\left(E I \Psi_{X}\right)_{X}-k A G\left(\Psi-W_{X}\right)-X\left(k A G\left(\Psi-W_{X}\right)\right)_{X} \\
& \quad-\rho\left(I+A X^{2}\right) \gamma(T) d X \\
= & {\left[E I \Psi_{X}-X k A G\left(\Psi-W_{X}\right)\right]_{0}^{L}-\gamma(T) \int_{0}^{L} \rho\left(I+A X^{2}\right) d X } \\
= & \tau+f L-\gamma(T) \int_{0}^{L} \rho\left(I+A X^{2}\right) d X \\
= & \left(\theta_{T T}-\gamma(T)\right) \int_{0}^{L} \rho\left(I+A X^{2}\right) d X .
\end{aligned}
$$

Hence the Lagrange multiplier $\gamma$ is identified as the angular acceleration:

$$
\gamma(T)=\theta_{T T}
$$

Substituting (34) into (31) yields

$$
\begin{aligned}
\rho A \tilde{W}_{T T}+\left(k A G\left(\tilde{\Psi}-\tilde{W}_{X}\right)\right)_{X} & =0, \\
\rho I \tilde{\Psi}_{T T}-\left(E I \tilde{\Psi}_{X}\right)_{X}+k A G\left(\tilde{\Psi}-\tilde{W}_{X}\right) & =0 .
\end{aligned}
$$

where $\tilde{W}=W+X \theta$ and $\tilde{\Psi}=\Psi+\theta$ as defined. Hence Equation (35) has been put in exactly the same form as that of (2) simply by replacing $W$ with the arc length $(W+X \theta)$ and $\Psi$ by $(\Psi+\theta)$ as shown in Figure 3.

The associated boundary conditions of the rotating beam in terms of $\tilde{W}$ and $\tilde{\Psi}$ are

$$
\begin{aligned}
\tilde{W}(0, T) & =0, \\
E I(0) \tilde{\Psi}_{X}(0, T) & =-\tau(T), \\
\tilde{\Psi}_{X}(L, T) & =0, \\
k A G(L)\left(\tilde{\Psi}(L, T)-\tilde{W}_{X}(L, T)\right) & =-f(T),
\end{aligned}
$$


which are identical to those of the hinged beam as given by Equations (11).

\subsection{Partial Boundary Controllability of the Rotating Beam}

Recall from Section 2.6 that the hinged beam can be driven to one of the states given by (15)

$$
W_{T}(X, \mathcal{T})=\Psi_{T}(X, \mathcal{T})=0, \quad \Psi(X, \mathcal{T})=\theta_{o}, \quad W(X, \mathcal{T})=\theta_{o} X
$$

where $\theta_{o}$ is a constant and $\mathcal{T}>2 \max \left(T_{1}, T_{2}\right)$ with $T_{1}$ and $T_{2}$ defined by Equations (14). Consequently the rotating beam can be driven to one of the states

$$
\begin{aligned}
& \tilde{W}_{T}(X, \mathcal{T})=\tilde{\Psi}_{T}(X, \mathcal{T})=0, \\
& \tilde{\Psi}(X, \mathcal{T})=\theta_{f}, \quad \tilde{W}(X, \mathcal{T})=\theta_{f} X,
\end{aligned}
$$

with $\theta_{f}=\theta_{o}+\theta_{\mathcal{T}}$ where $\theta_{\mathcal{T}}$ is the weighted average angular displacement of the beam at time $\mathcal{T}$.

The following conditions are relevant to our controllability results:

1. $\rho, A, I, k, G$ and $E$ are all positive functions of the space variable $X$ and all belong to $C^{2}([0, L])$.

2. $\mathcal{T}>2 \max \left(T_{1}, T_{2}\right)$.

We summarise these partial controllability results as a theorem.

Theorem 4 (Controllability) Suppose that conditions 1-2 above hold. Then the following statements are true. 
1. Suppose that there are no nontrivial solutions of the eigenvalue problem (16), (17). Given finite energy initial data of the hinged beam problem (2), (11) and (25), there exist control functions $f \in L^{2}(0, \mathcal{T})$ and $\tau \in L^{2}(0, \mathcal{T})$, that drive the system to its rest state at time $\mathcal{T}$ :

$$
\begin{aligned}
W(X, \mathcal{T})-\theta_{o} X=\Psi(X, \mathcal{T})-\theta_{o} & =0 \\
W_{T}(X, \mathcal{T})=\Psi_{T}(X, \mathcal{T}) & =0, \quad 0<X<L
\end{aligned}
$$

2. Suppose that there are no nontrivial solutions of the eigenvalue problem (16), (17). Given finite energy initial data of the rotating beam problem (35), (36) and (25), there exist control functions $f \in L^{2}(0, \mathcal{T})$ and $\tau \in L^{2}(0, \mathcal{T})$, that drive the system to its rest state at time $\mathcal{T}$ :

$$
\begin{aligned}
\tilde{W}(X, \mathcal{T})-\theta_{f} X=\tilde{\Psi}(X, \mathcal{T})-\theta_{f} & =0, \\
\tilde{W}_{T}(X, \mathcal{T})=\tilde{\Psi}_{T}(X, \mathcal{T}) & =0, \quad 0<X<L .
\end{aligned}
$$

with $\theta_{f}=\theta_{o}+\theta_{\mathcal{T}}$ where $\theta_{\mathcal{T}}$ is the weighted average angular displacement of the beam at time $\mathcal{T}$.

3. If there exist nontrivial solutions of the eigenvalue problem (16) and (17), then both the hinged problem (2), (11) and (25), and the rotating problem (35), (36) and (25), are not even approximately controllable. 


\subsection{Existence of Solutions to the Beam Equations}

For the system (35) and (36), we use $\tilde{\mathbf{U}}=\left[\tilde{U}_{1}, \tilde{U}_{2}, \tilde{U}_{3}, \tilde{U}_{4}\right]^{T}$ in the same way as in Section 2.7 and from (19)

$$
\begin{aligned}
& \tilde{U}_{1}=-\left(\sqrt{k A G}\left(\tilde{\Psi}-\tilde{W}_{X}\right)+\sqrt{\rho A} \tilde{W}_{T}\right) / 2, \\
& \tilde{U}_{2}=-\left(\sqrt{k A G}\left(\tilde{\Psi}-\tilde{W}_{X}\right)-\sqrt{\rho A} \tilde{W}_{T}\right) / 2, \\
& \tilde{U}_{3}=-\left(\sqrt{E I} \tilde{\Psi}_{X}-\sqrt{\rho I} \tilde{\Psi}_{T}\right) / 2, \\
& \tilde{U}_{4}=-\left(\sqrt{E I} \tilde{\Psi}_{X}+\sqrt{\rho I} \tilde{\Psi}_{T}\right) / 2,
\end{aligned}
$$

with Equation (20) now taking the form

$$
\tilde{\mathbf{U}}_{T}+\Lambda \tilde{\mathbf{U}}_{X}=B \tilde{\mathbf{U}}-\Lambda_{X} \tilde{\mathbf{U}} / 2 .
$$

The mechanical energy of the beam is in the same form as (21) and (22):

$$
\begin{aligned}
\tilde{\mathcal{E}} & =\frac{1}{2} \int_{0}^{L} k A G\left(\tilde{\Psi}-\tilde{W}_{X}\right)^{2}+\rho A \tilde{W}_{T}^{2}+E I \tilde{\Psi}_{X}^{2}+\rho I \tilde{\Psi}_{T}^{2} d X \\
& =\int_{0}^{L} \tilde{U}_{1}^{2}+\tilde{U}_{2}^{2}+\tilde{U}_{3}^{2}+\tilde{U}_{4}^{2} d X
\end{aligned}
$$

The boundary conditions analogous to (23) now take the form

$$
\begin{aligned}
\tilde{U}_{2}(0, T)-\tilde{U}_{1}(0, T) & =0 \\
\tilde{U}_{4}(0, T)+\tilde{U}_{3}(0, T) & =(E I)(0)^{-1 / 2} \tilde{\tau}(T), \\
\tilde{U}_{4}(L, T)+\tilde{U}_{3}(L, T) & =0 \\
\tilde{U}_{2}(L, T)+\tilde{U}_{1}(L, T) & =(k A G)(L)^{-1 / 2} \tilde{f}(T) .
\end{aligned}
$$

Also the system is completed by including the initial condition which is the same as (25)

$$
\tilde{\mathbf{U}}(X, 0)=\mathbf{g}(X)
$$


with the variation of $\theta$ given by (32).

Theorems 1-3 in Section 2.7 for the hinged beam are also valid for the rotating beam.

\section{An Auxiliary Problem and Boundary Controllability}

So far we have been able to make use of (partial) controllability results in [6] already proven for the hinged beam. To make further progress and show that the rotating beam is (completely) controllable, we modify the procedure followed in [6], which makes use of a certain auxiliary problem to prove controllability.

\subsection{Auxiliary Problem and Contraction Properties}

If the length of our hinged beam is extended from $(0, L)$ to $(-\infty, L)$ and a second semi-infinite beam is hinged at the free end, we obtain a system which is very useful when considering controllability of the original system. This system consists of two semi-infinite beams as shown in Figure 4 which represent what we call an auxiliary problem. The boundary conditions at $X=0$ and $X=L$ are

$$
W(0, T)=\Psi_{X}(L, T)=0 .
$$

Further, since there is no external torque applied at the origin and displacement of the two beams must be the same at $X=L$, we have

$$
\Psi_{X}\left(0^{-}, T\right)=\Psi_{X}\left(0^{+}, T\right), \quad W\left(L^{-}, T\right)=W\left(L^{+}, T\right) .
$$




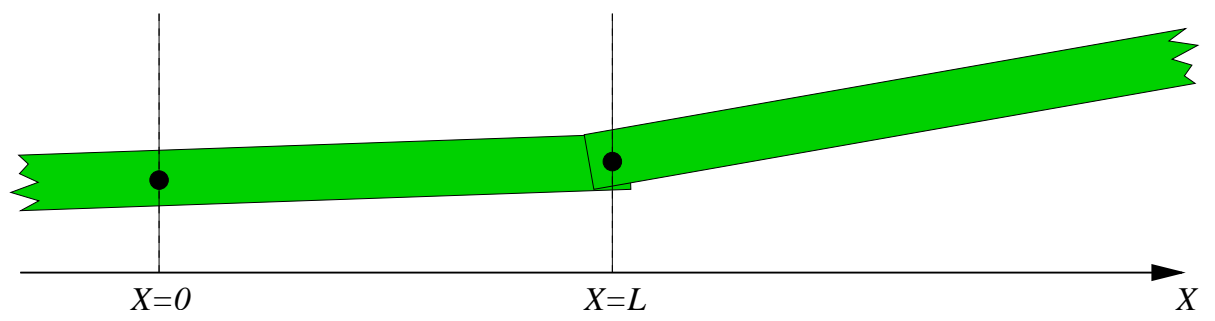

Figure 4: The Auxiliary Problem

Due to the above, it will be more convenient to break down the beam equation $\mathbf{U}_{T}+\Lambda \mathbf{U}_{X}=\mathbf{B U}-\Lambda_{X} \mathbf{U} / 2$ into two sub-equations for the auxiliary problem as follows:

$$
\begin{aligned}
& {\left[\mathbf{U}_{I}\right]_{T}+\Lambda_{I}\left[\mathbf{U}_{I}\right]_{X}=\mathbf{B}_{I} \mathbf{U}-\left[\Lambda_{I}\right]_{X} \mathbf{U}_{I} / 2,} \\
& \quad \text { for }(X, T) \in((-\infty, 0) \cup(0, \infty)) \times \mathbb{R} ; \\
& {\left[\mathbf{U}_{I I}\right]_{T}+\Lambda_{I I}\left[\mathbf{U}_{I I}\right]_{X}=\mathbf{B}_{I I} \mathbf{U}-\left[\Lambda_{I I}\right]_{X} \mathbf{U}_{I I} / 2,} \\
& \quad \text { for }(X, T) \in((-\infty, L) \cup(L, \infty)) \times \mathbb{R} ; \\
& U_{2}(0, T)-U_{1}(0, T)=0, T \in \mathbb{R} ; \\
& U_{4}(L, T)+U_{3}(L, T)=0, T \in \mathbb{R} ;
\end{aligned}
$$

where

$$
\begin{aligned}
& \mathbf{U}_{I}=\left[U_{1}, U_{2}\right]^{T}, \quad \mathbf{U}_{I I}=\left[U_{3}, U_{4}\right]^{T}, \quad \mathbf{U}=\left[\mathbf{U}_{I}, \mathbf{U}_{I I}\right]^{T} ; \\
& \mathbf{B}_{I}=\left[\begin{array}{rrrr}
0 & b_{1} & b_{2} & -b_{2} \\
-b_{1} & 0 & b_{2} & -b_{2}
\end{array}\right], \quad \mathbf{B}_{I I}=\left[\begin{array}{rrrr}
-b_{2} & -b_{2} & 0 & b_{3} \\
b_{2} & b_{2} & -b_{3} & 0
\end{array}\right], \\
& \mathbf{B}=\left[\begin{array}{l}
\mathbf{B}_{I} \\
\mathbf{B}_{I I}
\end{array}\right] ; \quad \Lambda_{I}=v_{1}\left[\begin{array}{ll}
1 & \\
& -1
\end{array}\right], \quad \Lambda_{I I}=v_{2}\left[\begin{array}{ll}
1 & \\
& -1
\end{array}\right], \\
& \Lambda=\left[\begin{array}{ll}
\Lambda_{I} & \\
& \Lambda_{I I}
\end{array}\right] ; \quad \mathbb{R}=(-\infty, \infty) .
\end{aligned}
$$

and $\left[\mathbf{U}_{I}\right]_{T},\left[\mathbf{U}_{I}\right]_{X}$ denotes the partial derivatives of $\mathbf{U}_{I}$ with respect to $T$ and $X$ respectively. 
For our controllability results we now assume that $\rho, E, G, k$, $A$ and $I$ are all positive, $C^{2}$ functions of the space variable. The properties of the auxiliary problem that we need for proving controllability of the rotating beam have already been proven in [6]. We summarise these below.

In the following theorems,

$$
\mathbf{U}(X, 0)=\mathbf{G}(X)=\left[G_{1}(X), G_{2}(X), G_{3}(X), G_{4}(X)\right]^{T}
$$

and $\mathcal{H}=\left(L^{2}(\mathbb{R})\right)^{4}$ is the finite energy space with norm

$$
\|\mathbf{U}\|=\left(\int_{-\infty}^{\infty}\left|U_{1}\right|^{2}+\left|U_{2}\right|^{2}+\left|U_{3}\right|^{2}+\left|U_{4}\right|^{2} d X\right)^{1 / 2}
$$

and $\mathcal{D}$ is the set of functions $U \in \mathcal{H}$ such that

1. $U_{1}$ and $U_{2}$ are in $\mathcal{H}^{1}(-\infty, 0)$ and $\mathcal{H}^{1}(0, \infty)$,

2. $U_{3}$ and $U_{4}$ are in $\mathcal{H}^{1}(-\infty, L)$ and $\mathcal{H}^{1}(L, \infty)$,

3. $U_{1}-U_{2}$ and $U_{3}+U_{4}$ are almost everywhere equal to continuous functions, and in this sense $U_{1}(0)-U_{2}(0)=0, U_{3}(L)+U_{4}(L)=$ 0 .

For Theorem(5), we let $\mathcal{B}$ be the operator on $\mathcal{H}$ with domain $\mathcal{D}$ given by

$$
\mathcal{B} \mathbf{U}=-\Lambda \mathbf{U}_{X}-\Lambda_{X} \mathbf{U} / 2+\mathbf{B U} .
$$

Theorem 5 (Finite Energy Solutions $I I) \mathcal{B}$ is the infinitesimal generator of a strongly continuous unitary group $\mathcal{U}(T)$ on $\mathcal{H}$. 
Of course, the fact that $\mathcal{U}(T)$ is unitary for each $T$ corresponds to conservation of energy for the physical system. The solution of the auxiliary problem with initial value $\mathbf{g}(X)$ is

$$
\mathbf{U}(X, T)=\mathcal{U}(T) \mathbf{g}(X) .
$$

Theorem 6 (Trace Property) The restrictions of components of finite energy solutions to lines parallel to the $T$-axis are locally $L^{2}$ functions. Moreover, if $\mathbf{U}$ is such a solution, then the mapping $X \rightarrow$ $U_{i}(X, \cdot)$ into $L_{\text {loc }}^{2}(\mathbb{R})$, is continuous everywhere except possibly at $X=$ 0 for (40) and $i=1,2$, and at $X=L$ for (40) and $i=3,4$. At these discontinuities, the left and right limits of the mapping exist.

Recall that boundary control of hyperbolic systems requires a time interval determined by the speed of the characteristics. In our case we must take into account the time it takes for disturbances to traverse the whole length $[0, L]$ of the physical beam twice, as is illustrated in Figure 5. In this figure, $C_{1}$ is the union of the characteristic with velocity $-v_{1}(X)$ starting at $X=L, T=0$ and the characteristic with velocity $v_{1}(X)$ starting at $X=0$ at the time when the former characteristic meets the $T$-axis. $C_{2}$ is a similar union of characteristics with velocities $\pm v_{2}(X)$. We set

$$
T_{0}=2 \max \left(\int_{0}^{L} \frac{d s}{v_{1}(s)}, \int_{0}^{L} \frac{d s}{v_{2}(s)}\right) .
$$

Let $\mathcal{S}$ be the subspace of $\mathcal{H}$ consisting of functions with supports in the interval $[0, L]$ and let $\mathbf{P}$ denote the projection onto $\mathcal{S}$. Thus $\mathbf{P}$ may be regarded as the operator which multiplies functions by the characteristic function of the interval $[0, L]$. 


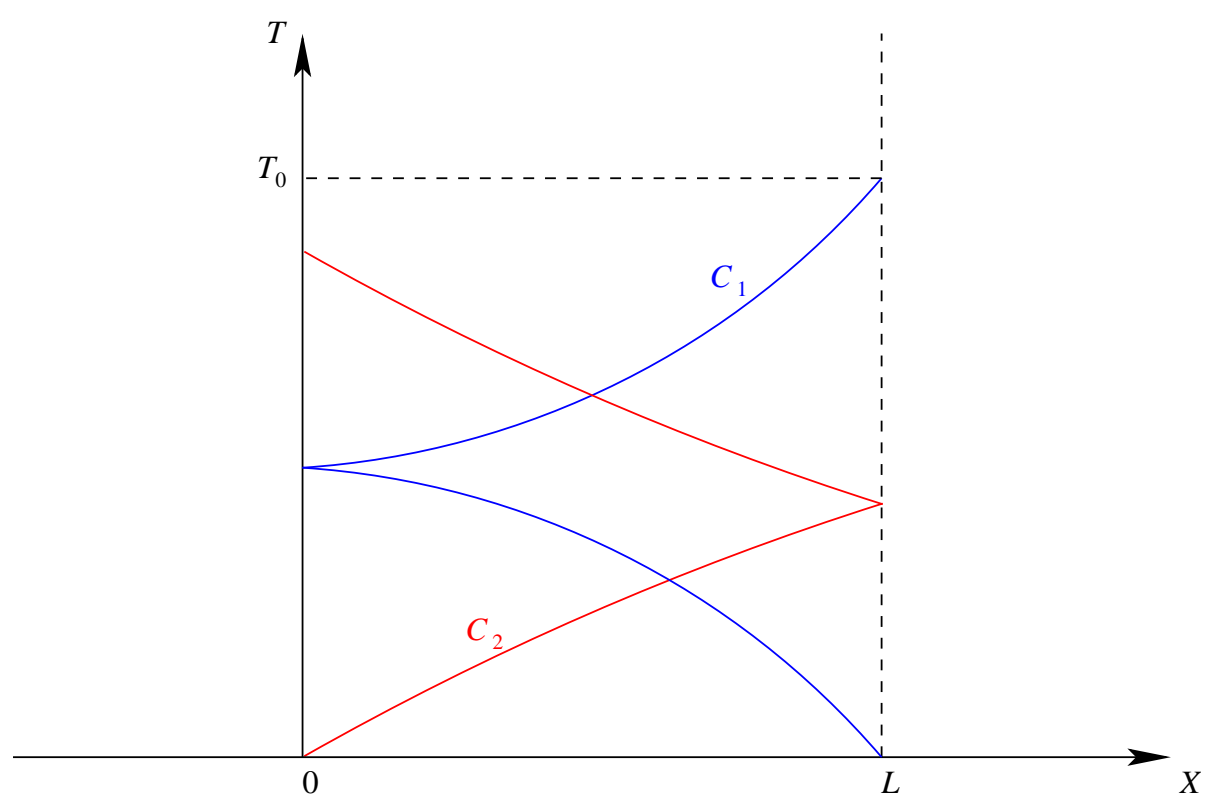

FiguRE 5: Relationship between $T_{0}$ and the characteristics 
1. $\mathcal{T}>T_{0}$

2. There are no non-trivial solutions of the over-determined eigenvalue problem (16)-(17);

3. The wave speeds $v_{1}(X)$ and $v_{2}(X)$ are distinct at each point $X \in[0, L]$

then $\|\mathbf{P} \mathcal{U}(\mathcal{T}) \mathbf{P}\|<1$.

The distinctness of the wave speeds is a technical requirement of some of the proofs in [6] and it is possible that it is not needed. However, according to the discussion of Section 2.4, real beams satisfy this requirement anyway.

\subsection{Controlling the Hinged Beam}

Before considering the control of the rotating beam, it is instructive to see how Theorem 4 for controllability of the hinged beam may be deduced from Theorem 7 .

The idea is to start with initial data for the boundary control problem and extend it to be initial data for the auxiliary problem by letting it be equal to zero outside the interval $[0, L]$. Let $\mathbf{g}$ denote the extension. Notice that $\mathbf{g} \in \mathcal{S}$. Next, we seek new initial data $\mathbf{f} \in \mathcal{H}$ such that:

1. $\mathbf{f}(X)=\mathbf{g}(X)$ a.e. for $X \in[0, L]$;

2. $\mathbf{U}(X, \mathcal{T})=0$ a.e. for $X \in[0, L]$, where $\mathbf{U}(., T)=\mathcal{U}(T) \mathbf{f}$. 
If we can find such initial data $\mathbf{f}$ then we can find appropriate control functions $f$ and $\tau$ for the boundary control problem by evaluating the boundary conditions (38) for the known solution $\mathbf{U}(X, T)$ of the auxiliary problem. Further, the trace property, Theorem 6 , shows that $f, \tau \in L^{2}(0, \mathcal{T})$.

It remains to see how we can find $\mathbf{f}$. The two conditions listed above for $\mathbf{f}$ may be written

$$
\begin{gathered}
\mathbf{P f}=\mathrm{g}, \\
\mathbf{P} \boldsymbol{\Phi f}=\mathbf{0}
\end{gathered}
$$

where $\Phi=\mathcal{U}(\mathcal{T})$. $\Phi$ is a unitary operator so $\Phi^{*}=\Phi^{-1}$, where $\boldsymbol{\Phi}^{*}$ and $\boldsymbol{\Phi}^{-1}$ denote the dual and inverse of $\boldsymbol{\Phi}$ respectively. We now see how to find such an $\mathbf{f}$ satisfying these properties and the additional property that $\|f\|$ is the smallest possible.

We attempt to do this using Lagrange multipliers $\gamma_{1}$ and $\gamma_{2}$ belonging to $\mathcal{H}$ and the functional

$$
\mathcal{J}(\mathbf{f})=\frac{1}{2}(\mathbf{f}, \mathbf{f})-\left(\mathbf{P f}-\mathbf{g}, \boldsymbol{\gamma}_{1}\right)-\left(\mathbf{P} \boldsymbol{\Phi} \mathbf{f}, \boldsymbol{\gamma}_{2}\right) .
$$

The first variation of $\mathcal{J}$ is

$$
\begin{aligned}
\delta \mathcal{J} & =\frac{1}{2}(\mathbf{f}, \delta \mathbf{f})+\frac{1}{2}(\delta \mathbf{f}, \mathbf{f})-\left(\mathbf{P} \delta \mathbf{f}, \boldsymbol{\gamma}_{1}\right)-\left(\mathbf{P} \mathbf{\Phi} \delta \mathbf{f}, \boldsymbol{\gamma}_{2}\right) \\
& =(\delta \mathbf{f}, \mathbf{f})-\left(\delta \mathbf{f}, \mathbf{P} \boldsymbol{\gamma}_{1}\right)-\left(\delta \mathbf{f}, \boldsymbol{\Phi}^{*} \mathbf{P} \boldsymbol{\gamma}_{2}\right) \\
& =\left(\delta \mathbf{f}, \mathbf{f}-\mathbf{P} \boldsymbol{\gamma}_{1}-\mathbf{\Phi}^{*} \mathbf{P} \boldsymbol{\gamma}_{2}\right) .
\end{aligned}
$$

The requirement that $\delta \mathcal{J}=0$ yields

$$
\mathbf{f}=\mathbf{P} \boldsymbol{\gamma}_{1}+\mathbf{\Phi}^{*} \mathbf{P} \boldsymbol{\gamma}_{2}
$$

Substituting (43a) into (42b) yields

$$
\begin{aligned}
\mathbf{P} \boldsymbol{\Phi}\left(\mathbf{P} \gamma_{1}+\mathbf{\Phi}^{*} \mathbf{P} \gamma_{2}\right) & =0 \\
\mathbf{P} \gamma_{2} & =-\mathbf{P} \boldsymbol{\Phi} \mathbf{P} \gamma_{1} .
\end{aligned}
$$


Substituting (43b) into (43a) yields

$$
\mathbf{f}=\mathbf{P} \gamma_{1}-\boldsymbol{\Phi}^{*} \mathbf{P} \boldsymbol{\Phi} \mathbf{P} \gamma_{1}=\left(\hat{\mathbf{I}}-\boldsymbol{\Phi}^{*} \mathbf{P} \boldsymbol{\Phi} \mathbf{P}\right) \mathbf{P} \gamma_{1},
$$

where $\hat{\mathbf{I}}$ denotes the $4 \times 4$ identity matrix.

Substituting (43c) into (42a) yields

$$
\begin{array}{r}
\mathbf{P}\left(\hat{\mathbf{I}}-\boldsymbol{\Phi}^{*} \mathbf{P} \boldsymbol{\Phi} \mathbf{P}\right) \mathbf{P} \gamma_{1}=\mathbf{g}, \\
\mathbf{P} \gamma_{1}=\left(\hat{\mathbf{I}}-\mathbf{P} \boldsymbol{\Phi}^{*} \mathbf{P} \boldsymbol{\Phi} \mathbf{P}\right)^{-1} \mathbf{g} .
\end{array}
$$

Substituting (43d) into (43c) yields

$$
\mathbf{f}=\left(\hat{\mathbf{I}}-\boldsymbol{\Phi}^{*} \mathbf{P} \boldsymbol{\Phi} \mathbf{P}\right)\left(\hat{\mathbf{I}}-\mathbf{P} \boldsymbol{\Phi}^{*} \mathbf{P} \boldsymbol{\Phi} \mathbf{P}\right)^{-1} \mathbf{g} .
$$

We suppose that the conditions of the contraction result, Theorem 7 , are valid. If this is the case then $\|\mathbf{P} \mathbf{\Phi P}\|<1$ and hence $\left\|\mathbf{P} \boldsymbol{\Phi}^{*} \mathbf{P} \mathbf{\Phi} \mathbf{P}\right\|<1$, so the inverse operator appearing in Equation (44) is well defined and bounded. It is easy to verify that $\mathbf{f}$ satisfies the required properties.

\subsection{Controlling the Rotating Beam}

The discussion above for the control of the hinged beam shows how the beam may be brought to rest during a finite time interval but it does not address the final angle of inclination of the beam. In this section we consider the additional constraint that the final angle of inclination $\theta$ is zero.

Differentiating Equation (30) with respect to $T$ yields

$$
\theta_{T}=\frac{\int_{0}^{L} \rho I \tilde{\Psi}_{T}+\rho A X \tilde{W}_{T} d X}{\int_{0}^{L} \rho\left(I+A X^{2}\right) d X} .
$$


Integrating this over the time interval $[0, \mathcal{T}]$ yields $\int_{0}^{\mathcal{T}} \int_{0}^{L} \rho I \tilde{\Psi}_{T}+\rho A X \tilde{W}_{T} d X d T=(\theta(\mathcal{T})-\theta(0)) \int_{0}^{L} \rho\left(I+A X^{2}\right) d X$, that is $\int_{0}^{\mathcal{T}} \int_{0}^{L}-\sqrt{\rho I}\left(\tilde{U}_{4}-\tilde{U}_{3}\right)+\sqrt{\rho A} X\left(\tilde{U}_{2}-\tilde{U}_{1}\right) d X d T=$ constant or

$$
\int_{0}^{\mathcal{T}}(\mathbf{Z}, \tilde{\mathbf{U}}) d T=\kappa
$$

where $\mathbf{Z}(X)=[-\sqrt{\rho A} X, \sqrt{\rho A} X, \sqrt{\rho I},-\sqrt{\rho I}]^{T}, \tilde{\mathbf{U}}=\left[\tilde{U}_{1}, \tilde{U}_{2}, \tilde{U}_{3}, \tilde{U}_{4}\right]^{T}$ and $(\mathbf{Z}, \tilde{\mathbf{U}})$ denotes the Hilbert space inner product of $\mathbf{Z}$ and $\tilde{\mathbf{U}}$. The constraint equation can be rewritten as

$$
\begin{aligned}
\int_{0}^{\mathcal{T}}(\mathbf{Z}, \tilde{\mathbf{U}}) d T & =\int_{0}^{\mathcal{T}}(\mathbf{Z}, \mathbf{P} \mathcal{U}(T) \mathbf{f}) d T \\
& =\left(\mathbf{Z}, \mathbf{P} \int_{0}^{\mathcal{T}} \mathcal{U}(T) d T \mathbf{f}\right) \\
& =(\mathbf{Z}, \mathbf{P} \boldsymbol{\Omega}(\mathcal{T}) \mathbf{f}) \\
& =\kappa .
\end{aligned}
$$

where $\tilde{\mathbf{U}}(T)=\mathbf{P} \mathcal{U}(T) \mathbf{f}$ is equal to the solution in the interval $[0, L]$ and is zero outside the interval and $\boldsymbol{\Omega}(\mathcal{T})=\int_{0}^{\mathcal{T}} \mathcal{U}(T) d T$.

The constraint problem for rotating beam now takes the form: minimise

$$
\|\mathbf{f}\|^{2}=(\mathbf{f}, \mathbf{f})
$$

subject to

$$
\begin{aligned}
\mathbf{P f} & =\mathbf{g}, \\
\mathbf{P} \mathbf{\Phi f} & =\mathbf{0}, \\
(\mathbf{P} \Omega \mathbf{f}, \mathbf{Z}) & =\kappa .
\end{aligned}
$$


Using Lagrange multipliers $\gamma_{1} \in \mathcal{H}, \gamma_{2} \in \mathcal{H}$ and $\gamma_{3} \in \mathbb{R}$, we have

$$
\begin{aligned}
\mathcal{J}(\mathbf{f}) & =\frac{1}{2}(\mathbf{f}, \mathbf{f})-\left(\mathbf{P f}-\mathbf{g}, \boldsymbol{\gamma}_{1}\right)-\left(\mathbf{P} \boldsymbol{\Phi} \mathbf{f}, \boldsymbol{\gamma}_{2}\right)-\gamma_{3}((\mathbf{P} \boldsymbol{\Omega} \mathbf{f}, \mathbf{Z})-\kappa) \\
\delta \mathcal{J} & =(\delta \mathbf{f}, \mathbf{f})-\left(\mathbf{P} \delta \mathbf{f}, \boldsymbol{\gamma}_{1}\right)-\left(\mathbf{P} \boldsymbol{\Phi} \delta \mathbf{f}, \boldsymbol{\gamma}_{2}\right)-\gamma_{3}(\mathbf{P} \boldsymbol{\Omega} \delta \mathbf{f}, \mathbf{Z}) \\
& =\left(\delta \mathbf{f}, \mathbf{f}-\mathbf{P} \boldsymbol{\gamma}_{1}-\mathbf{\Phi}^{*} \mathbf{P} \boldsymbol{\gamma}_{2}\right)-\gamma_{3}\left(\delta \mathbf{f}, \boldsymbol{\Omega}^{*} \mathbf{P Z}\right) \\
& =\left(\delta \mathbf{f}, \mathbf{f}-\mathbf{P} \boldsymbol{\gamma}_{1}-\boldsymbol{\Phi}^{*} \mathbf{P} \boldsymbol{\gamma}_{2}-\gamma_{3} \boldsymbol{\Omega}^{*} \mathbf{P Z}\right)
\end{aligned}
$$

But we require $\delta \mathcal{J}=0$, so we have

$$
\mathbf{f}=\mathbf{P} \gamma_{1}+\boldsymbol{\Phi}^{*} \mathbf{P} \gamma_{2}+\gamma_{3} \boldsymbol{\Omega}^{*} \mathbf{P Z}
$$

Substituting (46a) into (45a) yields

$$
\mathbf{P} \gamma_{1}+\mathbf{P} \boldsymbol{\Phi}^{*} \mathbf{P} \gamma_{2}+\gamma_{3} \mathbf{P} \boldsymbol{\Omega}^{*} \mathbf{P Z}=\mathbf{g} .
$$

Substituting (46a) into (45b) yields

$$
\mathbf{P} \gamma_{2}=-\left(\mathbf{P} \boldsymbol{\Phi} \mathbf{P} \gamma_{1}+\gamma_{3} \mathbf{P} \boldsymbol{\Phi} \boldsymbol{\Omega}^{*} \mathbf{P Z}\right) \text {. }
$$

Substituting (46c) into (46b) yields

$$
\mathbf{P} \gamma_{1}=-\left(\hat{\mathbf{I}}-\mathbf{P} \Phi^{*} \mathbf{P} \boldsymbol{\Phi} \mathbf{P}\right)^{-1}\left(\gamma_{3}\left(\mathbf{P}-\mathbf{P} \Phi^{*} \mathbf{P} \boldsymbol{\Phi}\right) \boldsymbol{\Omega}^{*} \mathbf{P Z}-\mathbf{g}\right)
$$

Substituting (46c) and (46d) into (46a) yields

$$
\begin{aligned}
\mathbf{f}= & \left(\hat{\mathbf{I}}-\boldsymbol{\Phi}^{*} \mathbf{P} \boldsymbol{\Phi} \mathbf{P}\right) \mathbf{P} \gamma_{1}+\gamma_{3}\left(\hat{\mathbf{I}}-\boldsymbol{\Phi}^{*} \mathbf{P} \boldsymbol{\Phi}\right) \boldsymbol{\Omega}^{*} \mathbf{P Z} \\
= & \gamma_{3}\left(\hat{\mathbf{I}}-\boldsymbol{\Phi}^{*} \mathbf{P} \mathbf{\Phi} \mathbf{P}-\left(\hat{\mathbf{I}}-\mathbf{P} \boldsymbol{\Phi}^{*} \mathbf{P} \boldsymbol{\Phi} \mathbf{P}\right)^{-1}\left(\mathbf{P}-\mathbf{P} \boldsymbol{\Phi}^{*} \mathbf{P} \boldsymbol{\Phi}\right)\right) \boldsymbol{\Omega}^{*} \mathbf{P Z} \\
& +\left(\hat{\mathbf{I}}-\boldsymbol{\Phi}^{*} \mathbf{P} \boldsymbol{\Phi} \mathbf{P}\right)\left(\hat{\mathbf{I}}-\mathbf{P} \boldsymbol{\Phi}^{*} \mathbf{P} \boldsymbol{\Phi} \mathbf{P}\right)^{-1} \mathbf{g} \\
= & \gamma_{3} \overrightarrow{\mathbf{a}}+\overrightarrow{\mathbf{b}} .
\end{aligned}
$$

Substituting (46e) into (45c) yields

$$
\begin{aligned}
\left(\mathbf{P} \Omega\left(\gamma_{3} \overrightarrow{\mathbf{a}}+\overrightarrow{\mathbf{b}}\right), \mathbf{Z}\right) & =\kappa \\
\gamma_{3}(\mathbf{P} \boldsymbol{\mathbf { a }}, \mathbf{Z}) & =\kappa-(\mathbf{P} \boldsymbol{\Omega} \overrightarrow{\mathbf{b}}, \mathbf{Z}), \\
\gamma_{3} & =\frac{\kappa-(\mathbf{P} \Omega \overrightarrow{\mathbf{b}}, \mathbf{Z})}{(\mathbf{P} \boldsymbol{\mathbf { a }}, \mathbf{Z})}
\end{aligned}
$$


Finally, Substituting (46f) into (46e) yields

$$
\mathbf{f}=\frac{(\kappa-(\mathbf{P} \Omega \overrightarrow{\mathbf{b}}, \mathbf{Z}))}{(\mathbf{P} \Omega \overrightarrow{\mathbf{a}}, \mathbf{Z})} \overrightarrow{\mathbf{a}}+\overrightarrow{\mathbf{b}}
$$

where the vectors

$$
\begin{aligned}
& \overrightarrow{\mathbf{a}}=\left(\hat{\mathbf{I}}-\boldsymbol{\Phi}^{*} \mathbf{P} \boldsymbol{\Phi}-\left(\hat{\mathbf{I}}-\mathbf{P} \boldsymbol{\Phi}^{*} \mathbf{P} \boldsymbol{\Phi} \mathbf{P}\right)^{-1}\left(\mathbf{P}-\mathbf{P} \Phi^{*} \mathbf{P} \boldsymbol{\Phi}\right)\right) \boldsymbol{\Omega}^{*} \mathbf{P Z} \\
& \overrightarrow{\mathbf{b}}=\left(\hat{\mathbf{I}}-\Phi^{*} \mathbf{P} \boldsymbol{\Phi} \mathbf{P}\right)\left(\hat{\mathbf{I}}-\mathbf{P} \Phi^{*} \mathbf{P} \boldsymbol{\Phi} \mathbf{P}\right)^{-1} \mathbf{g}
\end{aligned}
$$

The control functions $f$ and $\tau$ for the rotating beam are now found as in the last section for the hinged beam. Note that we have one extra condition for controllability: that $(\mathbf{P} \boldsymbol{\Omega} \overrightarrow{\mathbf{a}}, \mathbf{Z}) \neq 0$. Clearly this condition, which may be verified for specific beams, must be satisfied for our method to work. However, it is not known if the condition is necessary for boundary controllability.

\section{References}

[1] M. Géradin, D. Rixen, Mechanical Vibrations: Theory and Application to Structural Dynamics, Wiley, Masson, (1994) E167

[2] F. John, Partial Differential Equations, Applied Mathematical Sciences 1, Springer-Verlag, New York, 4th ed (1982). E155

[3] B. W. Leonard, B. Budiansky, On Travelling Waves in Beams, Natl. Adv. Comm. Aeron. Report 1173, 1-27 (1954) E155

[4] R. D. Mindlin, H. Deresiewics, Timoshenko's Shear Coefficient for Flexural Vibrations of Beams, 2nd U.S. Natl. Cong. Appl. Mech., 175-178 (1955) E156 
[5] A. Pytel, F. L. Singer, Strength of Materials Harper \& Rows, New York 4th ed (1987) E156

[6] S. W. Taylor, A smoothing Property of a Hyperbolic System and Boundary Controllability, Journal of Computational and Applied Mathematics 114 23-40, N.H. Elsevier (2000) E146, E147, E158, E162, E163, E173, E175, E178 